\title{
Traduire
}

Ine autre perspective sur la tataduction

Revue française de la traduction

$242 \mid 2020$

Passons au vert

\section{Métamorphoses physiques et traductologiques de l'énergie}

\section{Lucile Gailliard}

\section{(2) OpenEdition}

1 Journals

Édition électronique

URL : http://journals.openedition.org/traduire/1982

DOI : 10.4000/traduire.1982

ISSN : 2272-9992

Éditeur

Société française des traducteurs

Édition imprimée

Date de publication : 30 juin 2020

Pagination : 23-36

ISSN : 0395-773X

\section{Référence électronique}

Lucile Gailliard, « Métamorphoses physiques et traductologiques de l'énergie », Traduire [En ligne], 242 |

2020, mis en ligne le 15 juillet 2020, consulté le 21 décembre 2020. URL : http://

journals.openedition.org/traduire/1982 ; DOI : https://doi.org/10.4000/traduire.1982 


\section{Métamorphoses physiques et traductologiques de l'énergie}

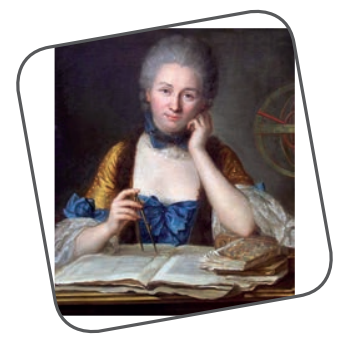

\section{Lucile Gailliard}

(C) Quentin de La Tour, Madame Du Châtelet à sa table de travail, XVIII ${ }^{\mathrm{e}}$ siècle ${ }^{1}$.

L'énergie est la "capacité d'un système à produire une activité externe ou à fournir un travail», nous dit l'Organisation internationale de normalisation [ISO, 2015]. Concrètement, qu'est-ce que cela signifie? Pourquoi devons-nous économiser ce qui, nous dit-on, se conserve? Pourquoi ce flou conceptuel, ces incohérences entre les sources - d'énergie et de terminologie? Qu'est-ce que l'énergie, exactement?

À l'heure de la transition énergétique, où plus un seul domaine ou presque de la traduction pragmatique ne peut s'affranchir d'un minimum de connaissances sur la question, nous nous proposons de remonter aux origines scientifiques et linguistiques de l'énergie; d'en asseoir la terminologie sur des bases physiques cohérentes; puis d'éclairer à la lumière de ces outils quelques difficultés de traduction récurrentes. 
Avertissement: la première occurrence des termes importants du domaine figure en gras. Les termes sont expliqués dans l'ordre dans lequel ils sont cités. La figure en fin d'article est une synthèse visuelle. Les exemples de traduction sont inspirés de cas réels mais, pour des raisons de confidentialité, ne sont pas des citations.

\section{Une brève histoire de l'énergie: et le mot rencontra le concept}

Quel est le point commun entre un ciel qui s'assombrit soudain, un éclair qui le zèbre, un feu de bois réconfortant, des châtaignes en train de cuire, et l'une d'elles, encore crue, roulant jusqu'à une autre?

Il fut un temps où la réponse était: "aucun». Nous avions une "conception substantielle des choses» [Klein, 2012], c'est-à-dire que nous constations, sans établir d'équivalence, la lumière (du soleil), l'électricité (de la foudre), la chaleur (d'une flamme), le mouvement (d'une châtaigne). Pourtant, la foudre peut arracher l'écorce d'un arbre, il faut d'autant plus de bois qu'on veut cuire de châtaignes, et un feu éclaire en même temps qu'il chauffe. À force d'observer des correspondances entre ces phénomènes, nous avons postulé l'existence d'une équivalence.

Petit retour en arrière. En 1687, quand Isaac Newton publie ses Philosophiae Naturalis Principia Mathematica, la communauté savante s'intéresse à la mécanique, c'est-à-dire au mouvement des corps. Qu'on pense à la châtaigne qui s'immobilise après avoir percuté sa voisine, tandis que celle-ci se met à rouler comme si la première lui avait cédé quelque chose: existe-t-il une formule mathématique pour calculer ce «quelque chose»? Partant du principe que le mouvement ne peut pas naître de rien, Newton fait l'hypothèse suivante: un corps qui en percute un autre lui fait gagner une quantité exactement égale à celle qu'il perd lui-même. Autrement dit, il existe une grandeur, formulable mathématiquement, qui se conserve au moment du choc: ce qui est perdu en amont est gagné en aval.

Que vaut cette grandeur? Newton postule que c'est le produit de la masse par la vitesse, ce que René Descartes a 
appelé «quantité de mouvement»². Outre-Rhin, Gottfried Wilhelm Leibniz défend pour sa part que c'est le produit de la masse par le carré de la vitesse, qu'il nomme "force vive». Cette contre-proposition est soutenue par la propre traductrice francophone de Newton, Émilie Du Châtelet, elle-même autrice de traités de physique. Pour trancher le dilemme, il faut conduire une expérience rigoureuse ${ }^{3}$ : celle-ci donnera raison, contre l'auteur, à la traductrice!

Pendant que le principe de conservation se clarifie en mécanique, on étudie la thermique ${ }^{4}$, c'est-à-dire la chaleur. Si des braises cuisent des châtaignes, est-ce parce qu'elles perdent une "quantité de chaleur» exactement égale à celle gagnée par les châtaignes? Osons même une passerelle entre les deux disciplines: si du feu produit de la vapeur qui produit du mouvement, peut-on calculer la quantité de feu nécessaire au mouvement voulu, en partant du principe que ce qui est perdu thermiquement est gagné mécaniquement? Les recherches s'accélèrent: machine à vapeur (James Watt, années 1770), pile électrique (Alessandro Volta, 1800), chaleur du rayonnement solaire (William Herschel, 1800), conversion de l'électricité ou de la force vive en chaleur (James Prescott Joule, années 1840). En 1845, Julius Robert von Mayer comprend aussi que la chlorophylle des plantes transforme la lumière en "différence chimique» [Balian, 2013]. En 1847 , Hermann Helmholtz conclut qu'avec la bonne formule mathématique on peut calculer l'équivalent thermique d'une force vive ou d'un rayonnement. Confondant cette grandeur avec une force, il la nomme lebendige Kraft, la force vive du français, traduite outre-Manche par le latin vis viva de Newton.

Mais en physique, la force recouvre une notion précise, or cette nouvelle grandeur n'est pas une force. Pour la nommer, Max Planck s'empare d'un mot issu du grec ancien, qui a longtemps vécu hors du champ de la physique où il n'est apparu sous sa forme française (sous la plume de

2. Quarante ans avant Newton, Descartes a fait la même hypothèse, mais dans une optique métaphysique: "Dieu aurait animé la matière et créé ainsi une certaine "quantité de mouvement", laquelle demeure conservée dans le temps.» [Gingras, 2018]

3. Willem Jacob's Gravesande mesure les enfoncements dans la glaise d'une boule de masse $3 \mathrm{~m}$ et de vitesse $v$, puis d'une boule de masse $m$ et de vitesse $3 v$, et observe un effet très supérieur dans le second cas [Balian, 2013].

4. On l'appelait alors calorifique. 
Jean Bernoulli, avec un sens restreint) qu'en 1717 et sous sa forme anglaise (dans les écrits de Thomas Young, avec un sens vague) qu'en 1807. En 1887, Planck publie Das Prinzip der Erhaltung der Energie et pose que la propriété fondamentale de l'énergie est de se conserver (erhalten, en allemand). L'énergie est un nombre qu'on peut calculer pour n'importe quel système et qui dit combien on peut espérer recevoir de lui sous chaque forme (manifestation physique): si un système cède de l'énergie à un autre, cet autre gagne exactement ce que le premier a perdu, sachant que l'énergie peut changer de forme au cours de l'échange - par exemple, de l'électricité dans le premier système peut se manifester sous forme de chaleur dans le deuxième.

L'énergie est donc une abstraction, une équivalence purement mathématique. Simple à transposer graphiquement dans de multiples langues européennes, elle est intraduisible, sans perte de sens, des mathématiques vers la langue elle-même: elle «met en crise les mots du langage parlé ${ }^{5}$ ». Pour qui doit non seulement écrire sans les mathématiques, mais aussi voyager d'une langue à l'autre, c'est un problème.

\section{Des ancrages physiques pour éviter les écueils terminologiques}

Parler d'énergie sans dire que c'est une abstraction est un abus de langage. Mais un abus de langage du point de vue physique en est-il un du point de vue linguistique quand il est entré dans l'usage? C'est dans ce contexte paradoxal que nous devons traduire. Pour faire le tri dans nos raccourcis de langue, (re)partons de la réalité physique.

\section{Entropie, transformation, chaleur fatale (perdue ou récupérée)}

L'énergie est une superhéroïne qui a sa superméchante. Toujours constante, elle est flanquée d'une autre grandeur, l'entropie, qui mesure l'incapacité d'un système à se transformer spontanément, et dont la propriété fondamentale

5. Expression employée par Étienne Klein [2018] à propos des mathématiques. 
est de toujours augmenter. Autrement dit, plus un système évolve, moins il peut évolver encore, sauf à puiser toujours davantage de ressources à l'extérieur de lui-même. Pour fixer les idées, passons par une analogie. Qui vous cède un roman perd un texte égal à celui que vous recevez: le transmettre n'en raccourcit pas la fin! Mais ce roman vous est cédé sur un support en papier condamné à s'user. Plus le roman passe de main en main, plus son usure augmente, même si le texte reste constant.

En vertu de ce principe, à chaque fois que de l'énergie change de forme - ce qu'on appelle une transformation -, une partie devient de la chaleur, dite fatale parce que ce sont les lois de la physique, et non la technique, qui lui imposent ce devenir. Par exemple, le fonctionnement d'un téléviseur est une transformation d'électricité en énergie radiative (lumière émise par l'écran), mécanique (vibrations de la membrane du haut-parleur) et thermique (chaleur fatale), quel que soit le modèle d'appareil. Les lois de la physique imposent à tout poste de télévision de «chauffer pour rien» l'air ambiant.

Pour ne pas perdre la chaleur fatale, on peut anticiper sa formation et, par exemple, inventer le poêle à bois: l'énergie d'un feu lancé pour cuire des châtaignes est transmise à l'air qui, au lieu de chauffer pour rien la cuisine (chaleur fatale perdue), se voit récupéré dans un tuyau pour chauffer des chambres à l'étage (chaleur fatale récupérée). Mais, quel que soit le procédé, de la chaleur fatale est toujours perdue: dans notre exemple, une partie de la chaleur du feu est transmise aux parois du poêle, puis à l'air ambiant.

C'est pourquoi il arrive toujours un moment où, malgré les progrès techniques, et même si nous avons récupéré les coques des châtaignes pour les brûler à leur tour, nous devons refaire provision de bois.

\section{Énergie, énergies, sources d'énergie primaires}

L'énergie, grandeur conservée, ne démarre nulle part ${ }^{6}$. Ce qui démarre quelque part, avec une source, c'est la chaîne de transformation mise en œuvre par l'être humain pour obtenir

6. Même pas de la matière puisque $E=m c^{2}$, mais c'est une autre histoire. 
des résultats (se chauffer, soulever une charge, se déplacer, etc.) avant la dégradation totale de l'énergie, c'est-à-dire sa dissipation ${ }^{7}$ en chaleur fatale perdue ${ }^{8}$. Une source d'énergie est donc un système qui contient (déjà) l'énergie dont nous avons besoin pour nos activités.

Dès lors, tout dépend de l'activité. Les batteries au lithium sont une source d'énergie pour les automobilistes, mais assurément pas pour les entreprises qui les fabriquent. De même, le fioul domestique (synonyme exact de mazout $^{9}$ ) et le fioul lourd sont des sources d'énergie pour les ménages et l'industrie, respectivement, mais aussi des produits du raffinage ${ }^{10}$.

Nous tendons, de ce fait, à vouloir remonter aux sources d'énergie primaires, c'est-à-dire directement issues de la nature. Nous extrayons l'énergie de la matière de trois façons: en la détruisant (uranium, biomasse); en exploitant son mouvement (air, eau); en puisant dans le flux d'énergie qu'elle transmet au milieu ambiant (soleil, croûte terrestre).

Pourquoi des énergies au pluriel si l'énergie en tant que telle est unique? Ce raccourci de langue aide à désigner les formes (mécanique, thermique, etc.) ou les origines (éolienne, solaire, etc.) de l'énergie. Mais, appliqué aux sources d'énergie elles-mêmes, il crée une incohérence logique puisqu'il confond matière exploitée (uranium, soleil) et forme d'énergie (radioactivité, rayonnement).

Pour corser l'affaire, certaines sources d'énergie (croûte terrestre, par exemple) sont assimilées à la forme d'énergie qu'elles délivrent (chaleur). On trouve donc des listes de sources d'énergie appelées "énergies» qui juxtaposent des matières (pétrole, océan), des flux de matière (vent) et des formes d'énergie (chaleur, radioactivité)!

7. L'usage veut qu'on emploie dégradation avec l'énergie et dissipation avec la chaleur ou l'électricité.

8. Perdue... pour l'activité humaine! À l'échelle de l'univers, cette forme d'énergie n'est ni plus ni moins perdue qu'une autre.

9. Mazout nous vient du russe, fioul de l'anglais.

10. C'est pourquoi la définition de «source d'énergie» varie selon les sources. 


\section{Sources d'énergie renouvelables, intermittentes}

Pour qu'une source d'énergie soit dite renouvelable, il faut que les conditions de son renouvellement soient à la mesure temporelle et spatiale de notre vie. Nous n'avons pas les moyens de patienter deux millions d'années (temps de formation du pétrole) ni de voyager jusqu'à une supernova (mort d'une étoile produisant des éléments radioactifs comme l'uranium). Inversement, nous n'avons pas à craindre la disparition du Soleil.

Répéter "provenant d'une source d'énergie renouvelable» serait indigeste, c'est pourquoi nous employons renouvelable dans ce sens également. En toute rigueur, une énergie renouvelable est une énergie tirée d'une source d'énergie renouvelable [ISO, 2015].

Une source d'énergie est également intermittente si elle ne nous est pas accessible en permanence (soleil, par exemple). Toutes les sources intermittentes sont renouvelables, mais l'inverse est faux (biomasse, par exemple).

\section{Électricité, énergie secondaire, centrale, réseau}

Nous savons extraire de la nature toutes les formes d'énergie sauf une: l'électricité. C'est pourquoi cette énergie est dite secondaire. Pour y accéder, nous devons procéder à une transformation dans une machine: un centre de production d'électricité s'appelle une centrale".

Comme nous ne savons pas stocker l'électricité ${ }^{12}$, nous avons dû créer des réseaux capables de l'acheminer, dès sa production, jusqu'aux points de consommation. Le secteur de l'électricité comprend donc: la production, avec un parc de centrales exploitées par des électriciens (ou exploitants); le transport, avec un réseau de lignes à haute tension; et la distribution, avec un réseau dédié qui approvisionne le public consommateur. Fournir de l'électricité au réseau se dit injecter. Prélever de l'électricité se dit soutirer. La mission consistant à vérifier que la quantité injectée est en perma-

11. Centrale électrique est un pléonasme.

12. Stocker "de l'électricité», c'est en fait emmagasiner de l'énergie sous une autre forme dans un système dont on pourra ultérieurement extraire de l'électricité: batterie (énergie chimique), station de transfert d'énergie par pompage (énergie mécanique) [CRE, 2020]. 
nence égale à la quantité soutirée incombe au gestionnaire de réseau de transport (GRT) [RTE].

\section{Puissance et loi de conservation de la puissance en français}

Pour anticiper la quantité d'électricité produite ou consommée par une machine, on calcule souvent sa puissance, c'està-dire ce qu'elle peut produire ou consommer par unité de temps. La puissance est exprimée en watts (W) ou ses multiples: kilowatts ( $k W$ ), mégawatts ( $M W$ ), gigawatts $(G W)^{13}$.

Dans une centrale thermique ${ }^{14}$, qu'elle soit à flamme (combustible carboné) ou nucléaire (combustible nucléaire), on distingue la puissance thermique en sortie de chaudière ${ }^{15}$ (watts thermiques ou Wth) de la puissance électrique en sortie d'alternateur (watts électriques ou We). Les lois de la physique limitent le rapport entre puissance électrique et puissance thermique, à cause de la chaleur fatale. C'est pourquoi la production combinée d'électricité et de chaleur (cogénération) est intéressante: de $40 \%$ environ, le rendement de la centrale peut passer à $90 \%$.

Loi de conservation de la puissance en français: toute grandeur exprimée en watts est une puissance, quel que soit le terme source!

\section{Translating energy: faux amis et vraies confusions}

Dans le domaine de l'énergie, l'étape de déverbalisation est souvent une étape de "physicisation»: quelles sont les sources, énergies, machines et transformations en jeu? Illustrons cette démarche avec quelques exemples.

13. Seul le k est en minuscule. Adressons un clin d'œil au personnage Emmett Brown du film Retour vers le futur, condamné en 1985 à parler de «gigowatts».

14. Pour suivre cette explication sur un schéma, voir par exemple EDF, Centrale à charbon.

15. Le réacteur d'une centrale nucléaire peut aussi être appelé chaudière. 
Application de la loi de conservation de la puissance en français

fusion power of $500 \mathrm{MW}$

puissance de fusion de $500 \mathrm{MW}$ $3000 \mathrm{MW}(\mathrm{th})$ reactor réacteur (d'une puissance) de 3000 MWth

$400 \mathrm{MW}$ thermal capacity

puissance de $400 \mathrm{MWth}$

$1 \mathrm{~kW}$ continuous thermal load

puissance continue de $1 \mathrm{~kW}$ th

developing at least $4 \mathrm{~kW}$

d'une puissance d'au moins $4 \mathrm{~kW}$

$500 \mathrm{MW}$ electric output

heat input of $800 \mathrm{MW}$

puissance de $500 \mathrm{MWe}$

puissance de 800 MWth

L'inventivité de l'anglais se heurte à la rigueur du français: une puissance est une puissance! Éventuellement, on choisira entre puissance thermique de $400 \mathrm{MW}$ - si public non spécialiste - et puissance de $\mathbf{4 0 0}$ mégawatts thermiques ( $M W$ th) - si public spécialiste et désir de ne pas répéter "thermique» dans la suite du texte ${ }^{16}$. Les nuances input et output (en entrée et en sortie) peuvent être nécessaires dans certains cas, mais pas en production d'électricité, où les lois de la physique dictent que la puissance thermique précède la puissance électrique.

\section{Quelques difficultés récurrentes}

Le terme fuel se traduit rarement en fonction d'un sens intrinsèque (voir aussi "Quand le terme répété n'est pas celui qui porte le sens»). À l'échelle du secteur énergétique, il peut désigner l'énergie en général (fuel poverty = précarité énergétique), les sources primaires par opposition à l'électricité (fuel mix for power generation = mix électrique), ou encore les combustibles par opposition aux autres sources (nonfossil fuel and renewables = sources d'énergie renouvelables et non fossiles).

Dans les transports, il désigne le carburant, que ce soit seul ou accompagné (transport fuel, motor fuel). Ironiquement, la traduction française d'expressions employant ce mot l'élude parfois: fuel station se dit station-service, fuel tourism se dit

16. En toute rigueur, puissance thermique de 400 MWth est un pléonasme. 
tourisme à la pompe (faire le plein, pour moins cher, dans le pays voisin), fuel technology est la technologie de motorisation et fuel economy... impose d'inverser le texte (voir "Quand improved fuel efficiency fait tout baisser»)!

Enfin, alors que fioul vient de fuel, c'est oil que l'on trouve dans la traduction de fioul domestique (heating oil) et fioul lourd (heavy oil), même si ce mot signifie pétrole dans le cas général des sources d'énergie fossiles (coal, gas, oil = charbon, gaz, pétrole).

Le terme power intervient souvent pour restreindre le champ étudié à celui de l'électricité (voir "Quand une apposition limite soudain le domaine»). Ainsi, nuclear energy (énergie nucléaire) est un hyperonyme de nuclear power (électronucléaire). Le nucléaire a aussi des applications non électrogènes (production d'hydrogène, par exemple).

Justement, ne pas confondre électrogène (qui produit de l'électricité) et électrique (qui consomme de l'électricité), parfois rendus en anglais par electric et electrical (electrical appliance = appareil électrique).

S'agissant de la production d'électricité, capacity désigne la puissance (installed capacity of a power plant = puissance installée d'une centrale). Il désigne une capacité uniquement quand il s'applique aux lignes à haute tension du réseau de transport (transmission capacities = capacités de transport). D'ailleurs, dans ce contexte, l'anglais transmission se traduit toujours par le français transport, ce qui nous amène à l'un des plus spectaculaires faux amis du domaine: a transmission system operator est un gestionnaire de réseau de transport.

En cas d'occurrence mystérieuse de capacities, dont on ne sait s'il s'agit d'une centrale, d'un parc de centrales, ou même de lignes à haute tension, on pourra opter pour le générique installations (the country plans to build new capacities = le pays prévoit de se doter de nouvelles installations).

\section{Quand le terme répété n'est pas celui qui porte le sens}

The potential for energy fuel switching within the energy system is unknown. Transport fuels and electricity prices are likely to increase, which would lead to a reduction in fossil fuel demand. 
But it is not known whether the distribution of primary energy supply by fuel type (fossil fuel, nuclear, renewables) would be affected.

Le passage démarre à l'échelle du système énergétique, ce que confirme le générique energy devant fuel. La présence de transport fait de transport fuels des carburants, tandis que l'inclusion de l'électricité fait de fossil fuel des combustibles. Enfin, comme la parenthèse qui suit type contient les sources non renouvelables et renouvelables, nous remontons au niveau logique des sources d'énergie primaires: profitonsen pour harmoniser (uranium, plutôt que nucléaire).

On ignore les possibilités de substitution au sein du mix énergétique. Les prix des carburants et de l'électricité vont probablement augmenter, ce qui ferait baisser la demande de combustibles fossiles. Mais rien ne dit que la répartition de l'offre primaire par source d'énergie (combustibles fossiles, uranium, sources renouvelables) serait modifiée.

\section{Quand une apposition limite soudain le domaine}

The availability of renewable energies is not always predictable. The computational model focuses on wind energy since it is the most widely deployed. However, it also applies to other intermittent renewable power source - i.e. solar and marine.

Cet extrait concerne les énergies renouvelables, distinguées selon leur origine. L'irruption de power à la troisième phrase réduit le domaine à la seule production d'électricité (donc ni éoliennes de pompage ni chauffe-eau solaires, notamment). Dans la version française, l'emploi de «centrale»épargnera la référence explicite à l'électricité, on pourra raccourcir intermittent renewable puisqu'une source intermittente est toujours renouvelable, et on se placera au niveau logique de la machine.

II n'est pas toujours possible de prévoir la disponibilité des centrales renouvelables. Le modèle de calcul cible les éoliennes, la technologie la plus déployée, mais s'applique aussi aux autres moyens de production intermittente - centrales solaires et marines. 


\section{Quand la diversité des niveaux logiques est un leurre}

Combustion engines may be replaced by battery vehicles or fuel cell technology.

Ne pas se laisser impressionner par les différences de niveau entre engine, vehicle et technology. Le domaine est le transport routier (vehicle), donc la machine est le moteur. La combustion est le seul moyen de transformer de l'énergie chimique en chaleur: moteur thermique et moteur à combustion interne sont synonymes. Au contraire, passer de l'énergie chimique à l'électricité peut se faire avec une batterie ou une pile à combustible.

Les moteurs thermiques pourraient être remplacés par des moteurs électriques à batterie ou à pile à combustible.

\section{Quand improved fuel efficiency fait tout baisser}

The fuel efficiency has greatly improved.

L'efficacité énergétique s'est-elle nettement améliorée? Dans le domaine du chauffage résidentiel, sans doute. Mais il y a une exception qui impose d'inverser tout le texte: le secteur automobile. En effet, la performance d'un véhicule (fuel efficiency ou fuel economy) est mesurée aux ÉtatsUnis en miles per gallon (distance parcourve pour un volume donné) et en France en litres pour cent kilomètres (volume consommé pour une distance donnée). Autrement dit, l'un des pays mesure l'inverse de ce que mesure l'autre. Dire à un lectorat francophone automobiliste qui «fait du 6 litres au 100 » que l'efficacité énergétique d'une voiture a augmenté, c'est oublier que la référence française est la consommation de carburant.

La consommation de carburant s'est notablement réduite.

\section{Conclusion}

La plus abstraite des grandeurs mathématiques est devenue la plus concrète de nos préoccupations. Les enjeux sont planétaires, les problèmes complexes, les réponses difficiles. 
Appuyons-nous sur la physique pour nous assurer, au moins, que nos textes sont aussi lisibles et cohérents que possible.

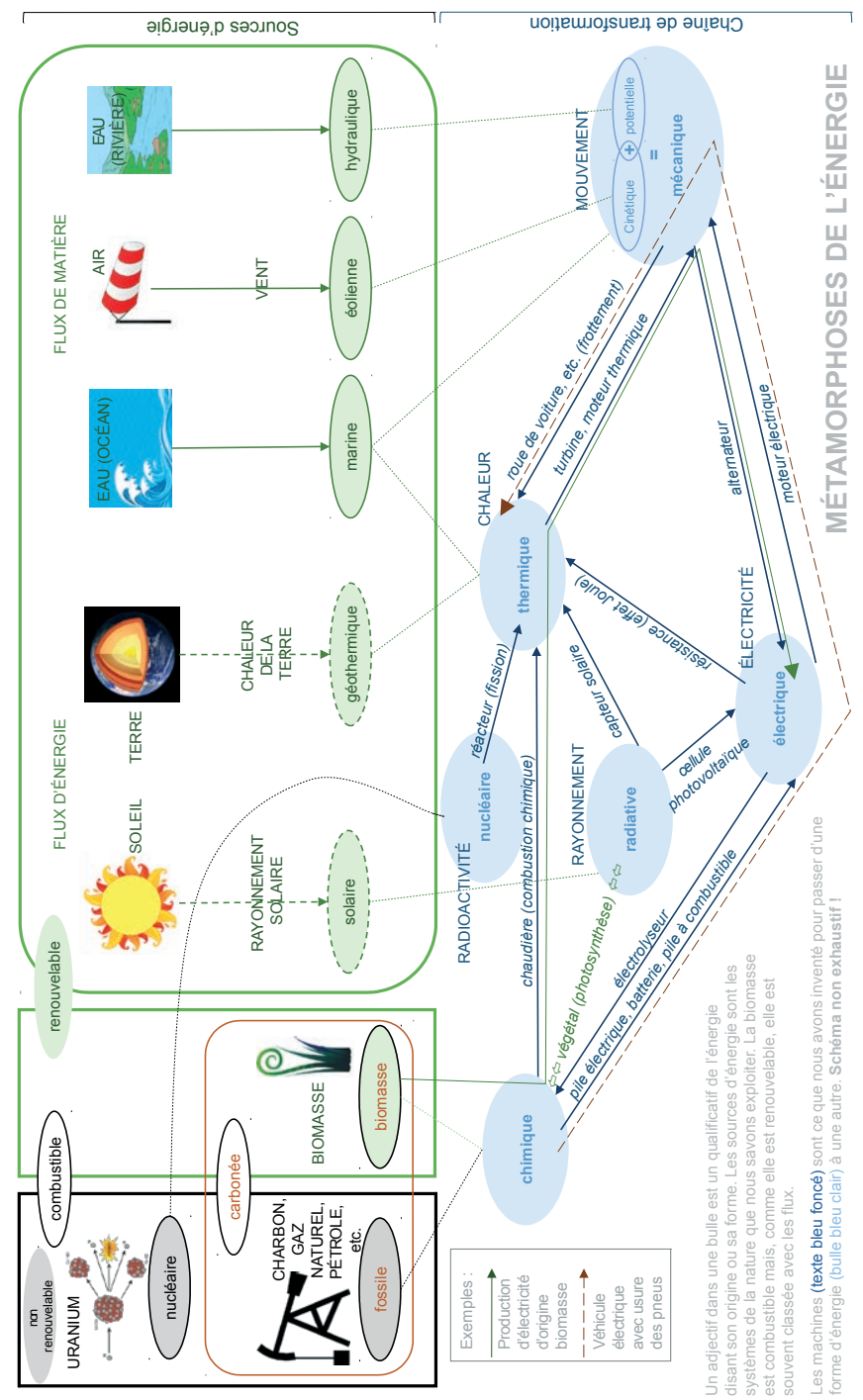

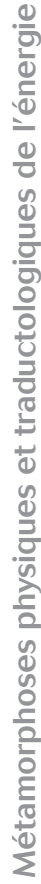

Figure 1. Métamorphoses de l'énergie. 
Lucile Gailliard est titulaire d'un diplôme d'ingénieur en génie thermique de l'École centrale de Marseille (France), d'un Master of Science en énergie appliquée de l'université de Cranfield (Royaume-Uni) et d'un master de traduction de I'université Paris VII (France). Après avoir exercé comme ingénieure développement dans l'industrie, elle a été traductrice indépendante spécialisée dans le domaine de l'énergie et animatrice d'ateliers de stylistique à l'université Paris VII. Elle est aujourd'hui traductrice dans une organisation internationale.

\section{SOURCES CITÉES}

Ademe, La chaleur fatale, Angers (France), Ademe, 2017, consulté le 20/05/2020.

BALIAN Roger, La longue élaboration du concept d'énergie, Paris, Académie des sciences, mars 2013, consulté le 20/05/2020. La première partie de cet article s'inspire principalement de cette source.

Commissariat à l'énergie atomique et aux énergies alternatives (CEA), Caractéristiques des diverses énergies, page web du CEA, juillet 2012, consulté le 20/05/2020.

Commission de régulation de l'énergie (CRE), Les différentes technologies stationnaires de stockage de l'électricité, page web SMARTGRIDS - CRE, consulté le 20/05/2020.

DU CHÂTELET (marquise) Gabrielle-Émilie Le Tonnelier de Breteuil, Institutions de physique, Paris, Chez Prault Fils, 1740, consulté le 20/05/2020.

Eurostat, glossaire de l'énergie en français et en anglais, consulté le 20/05/2020.

GINGRAS Yves, Histoire des sciences, Paris, Presses universitaires de France, coll. «Que sais-je?», 2018.

KLEIN Étienne, "De quoi l'énergie est-elle le nom?», cycle de conférences Cyclope, Paris-Saclay, CEA, 18 décembre 2012, consulté le 20/05/2020.

KLEIN Étienne, «La puissance du concept d'énergie», in Les Clés du CEA, 61, 2013, p. 4-6, consulté le 20/05/2020.

KLEIN Étienne, Matière à contredire, Paris, Éditions de l'Observatoire, 2018.

NEWTON Isaac, «par feue Madame la Marquise du Chastellet», Principes mathématiques de la philosophie naturelle, Paris, Desaint \& Saillant, 1759, consulté le 20/05/2020. C'est, à ce jour encore, la seule traduction française de l'œuvre de Newton.

Organisation internationale de normalisation (ISO), Efficacité énergétique et sources d'énergies [sic] renouvelables - Terminologie internationale commune - Partie 2: Sources d'énergie renouvelables, norme ISO/CEI 13273-2:2015, consulté le 20/05/2020.

Réseau de transport d'électricité (RTE), glossaire en français, consulté le 20/05/2020. 